\title{
Polarization behavior in systems with competing ferroelectric and antiferroelectric interactions
}

\author{
J. Milhazes, S. Lanceros-Méndez, and A. Cadilhe* \\ GCEP - Centro de Física da Universidade do Minho, 4710-057 Braga, Portugal
}

Received 7 September 2004, revised 15 November 2004, accepted 30 November 2004

Published online 2 February 2005

PACS 77.22.Gm, 77.80.Bh, 77.80.Dj

The dielectric hysteresis loop constitutes the main feature indicative of the properties of a ferroelectric material, but in complex systems with ferro- and antiferroelectric interactions, the hysteresis loops are often multiple. In the present work, we introduce a model based on the coexistence of ferro- and antiferroelectric regions, which can describe the aforementioned multiple loops. Based on our model, simulated multiple hysteresis loops are in good agreement with experimental results. Hysteresis loops obtained from our model are sensitive to the relative values of the ferro- and antiferroelectric coupling constants, domain size, and temperature. The model describes well systems without the presence of frustration as it is the case of $\mathrm{BA}_{x} \mathrm{BP}_{1-x}$. In the case of systems exhibiting frustration like e.g. $\mathrm{Rb}_{x}\left(\mathrm{NH}_{4}\right)_{1-x} \mathrm{H}_{2} \mathrm{PO}_{4}$, we present a straightforward extension of our model.

(C) 2005 WILEY-VCH Verlag GmbH \& Co. KGaA, Weinheim

\section{Introduction}

In a mixed ferro-(FE) and antiferroelectric (AFE) system, there is competition between the $\mathrm{FE}$ and the AFE orderings. Random distribution of composition is the main source to produce frustration, which can increase local structural competition such that the long-range electric order disappears. Instead of a typical sharp FE or AFE phase transition from the paraelectric (PE) phase, these systems are characterized by an extremely rich $x-\mathrm{T}$ phase diagram, where phase coexistence [such as PE/FE, PE/AFE, etc.] becomes a characteristic. In order to obtain locally a phase state, which is different from the disordered host, the minimal size of the short range ordered clusters must be big enough to show typical features of the longrange ordered phases.

There has been in the literature some effort to model ferroelectric materials, by using SherringtonKirkpatrick type of models, which can be analytically solved using mean-field methods [1]. More recently, the Transverse Ising Model, first proposed by P. G. de Gennes in 1963 [2], was shown to explain multiple hysteresis loops. Specifically, thin slabs of different ferroelectric strengths, with an interfacial antiferroelectric interaction separating sets of slabs, exhibits the above hysteresis loops [3]. This constitutes a significant improvement in our understanding of how ferroelectric materials behave. In this paper, we present theoretical results of a model based on FE clusters in an AFE matrix, capable of producing multiple hysteresis loops, and we show qualitative agreement with experimental results. Based on our model, we simulated single, double, and multiple hysteresis loops corroborated by experimental results. Comparison with experimental results is sensitive to the relative values of the coupling constants, grain size, and temperature. The phase diagram of the mixed crystal system Betaine Arsenate/Betaine Phosphate, to which a pseudo-spin model was applied, shows a rich variety of coexistence regions [4-6].

"Corresponding author: e-mail: cadilhe@fisica.uminho.pt 
The paper is organized as follows: In the following Section we present experimental results. In Section 3, we show the model and describe the details of simulations. In Section 4, the results are discussed. Finally, Section 5 is devoted to conclusions.

\section{Experimental}

The system chosen for the application of the model is the Betaine Arsenate/Betaine Phosphate mixed crystal system. Betaine arsenate $(\mathrm{BA}),\left(\mathrm{CH}_{3}\right)_{3} \mathrm{NCH}_{2} \mathrm{COO} \mathrm{H} \mathrm{AsO}_{4}$, which behaves ferroelectrically and betaine phosphate $(\mathrm{BP}),\left(\mathrm{CH}_{3}\right)_{3} \mathrm{NCH}_{2} \mathrm{COO} \mathrm{H}_{3} \mathrm{PO}_{4}$, which behaves antiferroelectrically are structurally closely related and mixed crystals are formed accordingly: $\mathrm{BA}_{x} \mathrm{BP}_{1-x}$, with $0 \leq x \leq 1$. The $(x, T)$-phase diagram of $\mathrm{BA}_{x} \mathrm{BP}_{1-x}[4-10]$ shows $\mathrm{FE}$ and $\mathrm{AFE}$ regions together with a variety of mixed regions in the intermediate concentration region. Though the phase diagram of the mixed $\mathrm{BA}_{x} \mathrm{BP}_{1-x}$ crystal system shows dielectric dispersion due to several relaxation processes at low temperatures, the typical features of the dipole glasses, such as the $\mathrm{Rb}_{x}\left(\mathrm{NH}_{4}\right)_{1-x} \mathrm{H}_{2} \mathrm{PO}_{4}$ [11] and the Betaine Phosphate/Betaine Phosphite (BPI) $\left(\mathrm{BP}_{x} \mathrm{BPI}_{1-x}\right)$ [12] mixed-crystal systems are absent in this system $[4,6]$. Further, the nature of the different mixed phase regions, transitions and relaxations of the phase diagram that reveal themselves in the measurements of the temperature and frequency dielectric function is still unclear and difficult to explain from the obtained structural $[13,14]$ and spectroscopic data [4].

Crystal structures of both BP [15] and BA [16] are quite similar and characterized by the existence of quasi-one dimensional chains of arsenate or phosphate tetrahedra, interconnected by hydrogen bonds. One betaine molecule is attached to each tetrahedron by two $H$-bonds via the carboxyl groups, which differ in strength in the case of BP but have approximately equal strength in BA. As a consequence, the deformations of the tetrahedra are subtly different in both compounds, resulting in their slightly different steric behavior. The length $\delta H$ of the $H$-bonds interconnecting the tetrahedra is almost identical in both compounds and $\delta H$ lies within the narrow region, where double-minimum potentials are to be expected for $H$-bonds. The single chains in both BA and BP are ferroelectrically ordered, the interchain interaction, however, slightly differs in both crystals, resulting in the FE order of BA and the AFE order in BP.

On the other hand, BP and BA are not isostructural with each other at room temperature $[15,16]$. BP undergoes a phase transition at $365 \mathrm{~K}$ from a paraelectric $\left(P 2_{1} / m(Z=2)\right.$ to an antiferrodistortive $\left(P 2_{1 / c}(Z=4)\right)$ phase and a further isostructural transition into an AFE phase at $T=86 \mathrm{~K}$, without doubling of the unit cell; for $T<81 \mathrm{~K} \mathrm{BP}$ is $\operatorname{AFE~}\left(P 2_{1} / c(Z=8)\right)[15,17]$. BA undergoes a phase transition from a paraelectric $\left(P c_{\mathrm{nm}}(Z=4)\right.$ high-temperature phase to a ferroelastic $\left(P 2_{1 / n}(Z=4)\right)$ one at $411 \mathrm{~K}$, and a further transition into a $\mathrm{FE}\left(P_{c}(Z=4)\right)$ phase at $T=119 \mathrm{~K}[16,18]$.

From dielectric measurements [4-10], the concentration-temperature diagram $((x, T)$-diagram) was divided into four different regions as indicated by the anomalies in $\mathcal{E}^{\prime}(T)$ and $\tan \delta(T)$ (i.e., $\mathcal{E}^{\prime \prime}(T)$ ). A $\mathrm{BP}$ region up to a concentration of $x=0.15$ and a BA region for concentrations larger that $x=0.80$ were defined. For these regions the behavior is essentially similar to the behavior of the two pure compounds, respectively. On the other hand, the intermediate regions $(0.15<x<0.80)$ are characterized by two anomalies in $\mathcal{E}^{\prime}(T)\left(T_{c 2, a}\right.$ and $\left.T_{c 2, b}\right)$ and $\tan \delta(T)\left(T_{d, a}\right.$ and $\left.T_{d, b}\right)$ 2. The shape and relative dielectric strength of these anomalies change with concentration and with external parameters such as pressure and/or bias field.

Regarding the hysteresis loops, the BP region is characterized by the typical double hysteresis loops below the AFE transition temperature, whereas in the BA-region single loops below the FE transition temperature are observed. This indicates the antiferroelectric and ferroelectric nature of these two regions of the phase diagram. In both cases the AFE or FE loops are observed down to the lowest temperatures and for the different concentrations within these regions, i.e., the nature of the phase does not seem to change neither with temperature nor concentration $[4,6]$.

On the other hand, within the intermediate regions the competition between AFE and FE interactions is larger, which in turn offers more complex hysteresis loops patterns both by varying concentration (the relative weight of the AFE and FE interactions) or temperature. 
$80.03 \mathrm{~K}$

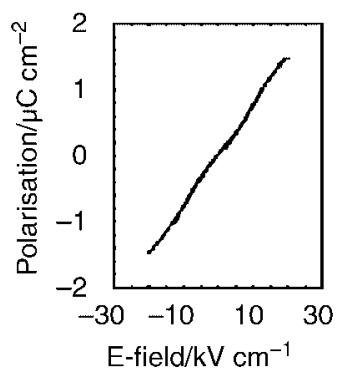

$67.12 \mathrm{~K}$

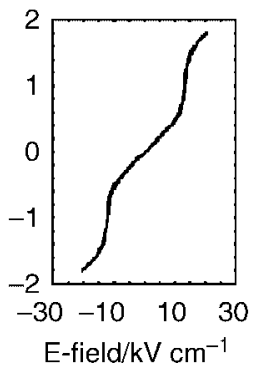

$50.14 \mathrm{~K}$

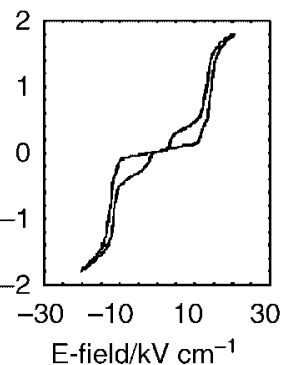

$34.44 \mathrm{~K}$

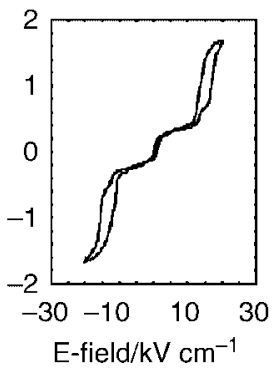

$24.91 \mathrm{~K}$

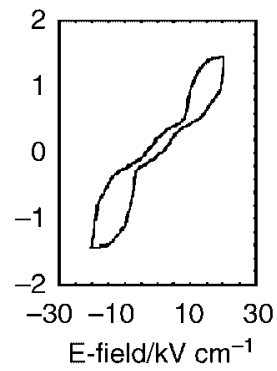

Fig. 1 Typical hysteresis loops for $\mathrm{BA}_{073} \mathrm{BP}_{027}$ at $p \sim 350 \mathrm{MPa}$ in different temperature regimes of the anomaly diagram (maximum field $E_{\max }=20.3 \mathrm{kV} \mathrm{cm}^{-1}$, measuring frequency $v=10 \mathrm{~Hz}$, sinusoidal field variation). For orientation purposes, the various anomaly temperatures are given: $T_{c 2} \sim 82 \mathrm{~K}, T_{c 2, b} \sim 60 \mathrm{~K}$.

As an example, the behavior of the $\mathrm{BA}_{0.73} \mathrm{BP}_{0.27}$ samples is shown in Fig. 1 [5]. This sample is representative of the existence of several contributions to the polarization, the complicated hysteresis loops and the strong changes under variation of temperature and external pressure. At $T_{c 2, a}$ a transition into an AFE ordered phase occurs at normal pressure (see Fig. 2). By increasing pressure, in the region in which the lower temperature anomaly in $\mathcal{E}^{\prime}(T)$ appears at $T_{c 2, b}$, the AFE hysteresis loops split into at least two AFE loops indicating two different dipolar systems. For decreasing values of the temperatures, the widths of the loops grow and those at low fields finally merge near $40 \mathrm{~K}$ to form a field induced lowtemperature FE phase, while the AFE loop at higher field strength persists (see Fig. 1.).

This behavior seems to reflect the fact that either a new collectively ordering dipole system appears or, more probably, that the original dipole system splits into two separately ordering parts. There is obviously a tendency that the original system maintains, at least partially, its AFE order, whereas the new system tends to develop a remanent polarization at lower temperatures [5], thus carrying pseudo-FE character. The onset of the remanent polarization is, however, not accompanied by an anomaly in $\mathcal{E}^{\prime}(T)$, thus it concerns a feature based essentially on the dipole relaxation. The contributions of the two dipolar systems to the spontaneous polarization remain roughly independent of temperature (Fig. 1) and pressure (not shown). On the other hand, in the region of the second critical pressure $\left(150 \mathrm{MPa}<p_{c 2}<250 \mathrm{MPa}\right)$ [5], where the second peak in $\tan \delta(T)$ (i.e., $\varepsilon^{\prime \prime}(T)$ ) appears, (Fig. 2) the corresponding polarization and current curves show that the higher temperature anomaly in $\tan \delta(T)\left(T_{d, a}\right)$ indicates the onset of a remanent polarization in the second pressure-induced dipole system. It becomes clear in the current loops where the corresponding relaxation peak passes approximately at $T_{d, a}$ the zero field axis. At intermediate pres-
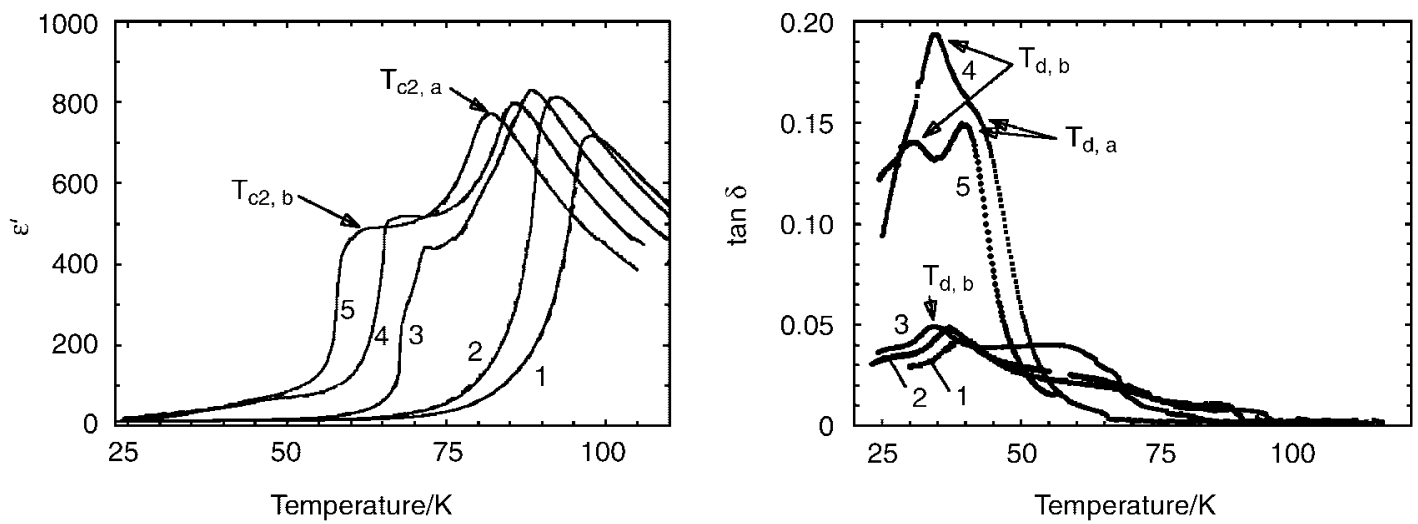

Fig. $2 \varepsilon^{\prime}(T)$ (left) and the corresponding $\tan \delta(T)$ curves (right) of $\mathrm{BA}_{0.73} \mathrm{BP}_{0.27}$ taken for several pressures and a measuring frequency $v=100 \mathrm{kHz}$ on cooling. $1: p=11-8 \mathrm{MPa} ; 2: p=75-60 \mathrm{MPa} ; 3: p=162-143 \mathrm{MPa}$; 4: $p=262-237 \mathrm{MPa} ; 5: p=353-343 \mathrm{MPa}$. 
sures $\left(p_{c 1}<p=150 \mathrm{MPa}<p_{c 2}\right)$, the features are much more complicated (being a mixture of the two cases before), so the enhancement in $\tan \delta(T)$ at $T \sim 60 \mathrm{~K}$ is also indicative of a similar change in the dipole system as at higher pressures.

In conclusion, the multiple shape of the hysteresis loops show the evolution of the different components of the polarization and its value in the different regions of the phase diagram. In particular, in the intermediate regions the coexistence of different AFE components or AFE and FE components is evidenced.

The quasi-one-dimensional Ising model has been applied to explain the behavior of the dielectric constant in the paraelectric phase [6] and dimensionality crossover from one to three dimensions by lowering the temperature has been reported [19]. Relaxational processes have been described in terms of "complex" and "simple" relaxations based on calculations of activation entropies [10, 20]. The nature of the relaxations (dipole-dipole and dipole-lattice interaction) was discussed as well as the variations induced by different external conditions. On the other hand, no theoretical model has been applied that account for the complexity of the different coexistence regions demonstrated by the multiple hysteresis loops.

\section{Model and simulations}

Ferroelectric materials with ferroelectric domains coupling antiferroelectrically with each other exhibit complex hysteresis loops, like ferroelectric, antiferroelectric, and mixed cases. To explain the complex behavior appearing in the hysteresis loops, we introduce the hamiltonian,

$$
\mathcal{H}=-\sum_{i=1}^{N} J_{i} \sum_{\langle i, j\rangle} s_{i} s_{j}+H \sum_{i=1}^{N} s_{i},
$$

where $J_{i} \in\left\{J_{\mathrm{FE}}, J_{\mathrm{AFE}}\right\},\langle i, j\rangle$ represents a sum over nearest neighbors, $N$ the number of pseudo-spins present in the lattice, $s_{i}$ are the pseudo-spins representing the dipole-dipole interactions, the subscripts FE and AFE are the ferroelectric and antiferroelectric couplings, and $H$ is the applied electric field, respectively. We assume that regions coupling ferroelectrically form domains, while regions coupling antiferroelectrically are at the interfaces between two ferroelectric domains. Real domains can take more complex geometries, different coupling strengths for interfacial and bulk interactions, different thicknesses of the interfacial walls separating different domains, and different domain sizes. In fact, it is possible to have materials with domains of pseudo-spins coupling ferroelectrically and antiferroelectrically. However, in the present study we show a robust mechanism, which can explain ferro- and antiferroelectric hysteresis loops in ferroelectric materials with competing ferroelectric and antiferroelectric interactions. Further, the mechanism, itself, is independent of the fact that our model is two-dimensional. In the case of a three dimensional model the values of the interaction constants change (the domain walls will be surfaces instead of lines), but the complex hysteresis loops remain a characteristic of the model. Ultimately, the different values of $J\left(J_{\mathrm{FE}}\right.$ and $\left.J_{\mathrm{AFE}}\right)$ affects the time scales of the processes involving coarsening and nucleation of clusters inside a particular domain. We define a parameter $\alpha=J_{F} / k_{B} T$ describing the ferroelectric coupling and thermal energy. The antiferroelectric coupling strength is defined in relation to the ferroelectric coupling as $\gamma=\left|J_{\mathrm{AF}} / J_{F}\right|$. For the sake of simplicity, we take a square lattice with square domains coupling antiferroelectric at the interface as shown in Fig. 3.

We performed a series of Monte Carlo simulations where we varied the applied electric field in time in order to observe the hysteresis loops for different domain sizes. Specifically, we obtained the hysteresis loops for domains of size 2 to 7, varied $\alpha$ between 0.4 to 2.5, and $\gamma$ between 1.0 to 4 (see Figs. 4 to 6). We executed a series of simulations for specific values of two of the parameters, $\alpha, \gamma$, and domain size, while varying the third one. The system is made of $10 \times 10$ domains in size, so, e.g., a system of domain size 5 will have a linear dimension of 50 sites. In each simulation, we carried out 1000 hysteresis loops. Though some finite size effect deviation from the thermodynamic limit in the simulated systems is expected, we do not foresee a strong size dependence of the various quantities we measured, so this effect is not considered in this preliminary study. 


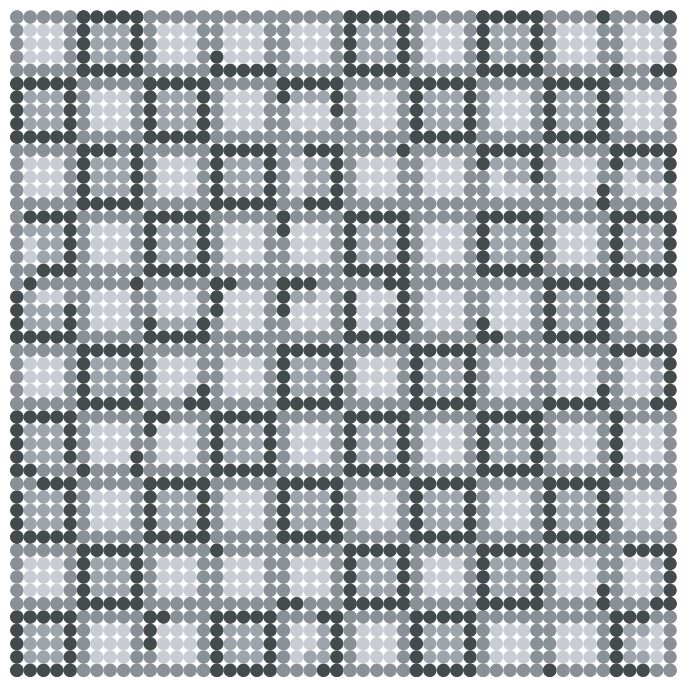

Fig. 3 Snapshot of the model with a domain size of 5 sites, and for $\alpha=1$ and $\gamma=1$. We notice that the saturation field is $E_{\text {Sat. }} \simeq 5$. Up pseudo-spins are represented in light grey in the bulk, while down spins are represented by a darker grey shade. Black represents down pseudo-spins interacting antiferroelectrically with pseudo-spins of a neighboring domain. Likewise, darkest grey shade represents the up pseudo-spins also interacting antiferroelectrically with pseudo-spins of a neighboring domain. In this particular snapshot, the external electric field is $E=0.736$ with a polarization of $P=-0.184$. The negative value of $P$ and positive value of $E$ is indicative of a rising magnitude and orientation in the positive direction of the electric field.

In Fig. 4, we present a tailored series of simulations for values of $\alpha$ of $0.4,1.0$, and 2.5, for a linear size of the domain of 6 , and for a $\gamma$ value of 4.0. At $\alpha=0.4$ the system is purely ferroelectric, but as we increase $\alpha$ (decrease the temperature at fixed coupling constant) to 0.7 , an antiferroelectric component superimposes. Further increase of the value of $\alpha$ tends to square the "elbows" appearing in the plot for $\alpha=0.7$. At $\alpha=2.5$, it becomes evident that segments of the hysteresis loop where the magnitude of the electric field raises are different from the ones with a decreasing electric field. Specifically, lowering the electric field leads to more abrupt changes of the polarization than in the case of a raising field.

In Fig. 5, we present the dependence on $\gamma$. At $\gamma=1.0$ and 1.5 the system is clearly ferroelectric, while for values around 2.0 and 2.5 the system develops instabilities, which for a value of $\gamma$ of 4.0 , antiferroand ferroelectric loops coexist.

In Fig. 6, we show that for a domain size of 3 the system is purely antiferroelectric. Moreover, complex antiferroelectric behavior is still present for domain sizes of 4 and 5. For a domain size of 6 , we observe coexistence of ferro- and antiferroelectric behavior. The ferroelectric hysteresis loop broadens with increasing domain size, as it can be observed for a domain size of 7 .

\section{Discussion}

The main issue of the present work was to discuss the relevant interactions behind the systems with complex ferroelectric and antiferroelectric behavior, with particular attention to those giving rise to multiple hysteresis loops. The theoretical model includes ferro- and antiferroelectric interactions in a particular way: ferroelectric clusters are embedded in an antiferroelectric matrix. As a consequence, the system shows paraelectric, simple ferroelectric, simple antiferroelectric or mixed loops depending on the relative size of the ferroelectric and antiferroelectric interactions, size of domains, and temperature (Figs. 4 to 6). Frustration stems from shared domain interface interaction of a domain with at least two other domains, while separated by antiferroelectric walls. Simulation results are representative of the behavior of the mixed crystal system $\mathrm{BA}_{x} \mathrm{BP}_{1-x}$, despite de simple two-dimensional model we used. This system shows the transition in concentration from simple antiferroelectric loops to simple ferroelectric while increasing the amount of the ferroelectric compound (BA). In the intermediate region no trace of frustration is found [4, 6], such as in similar systems like KDP/ADP [11] or BP/BPI [12], but the coexistence of multiple hysteresis loops. By tuning in an appropriate way the parameters of the model, the experimental (theoretical) loops can be simulated, as can be followed by comparison Figs. 1 and 7. After the model, the explanation of the existence of complex hysteresis loops becomes clear: as the amount of ferroelec- 

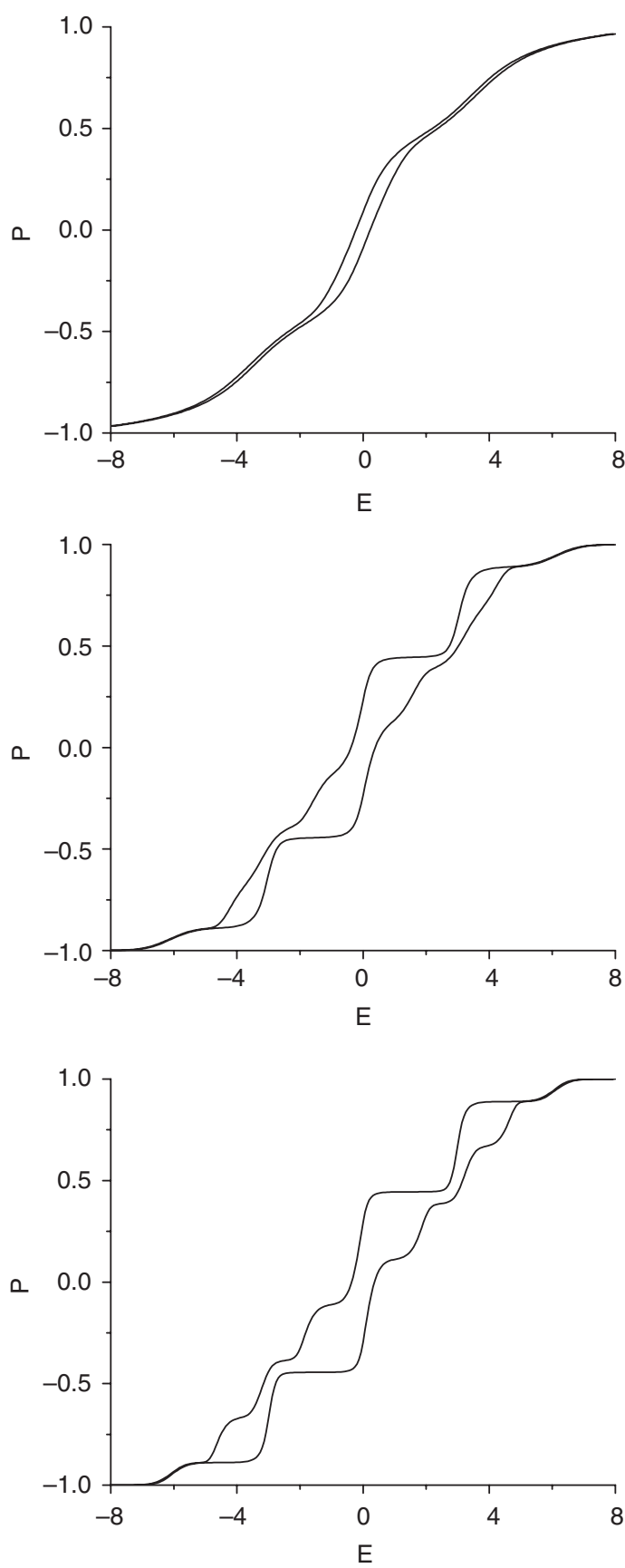

Fig. 4 Tailored series of hysteresis loops for values of $\alpha$, top to bottom, of $0.4,1.0$, and 2.5 for a linear size of the domain of 6 , and for a $\gamma$ value of 4.0 .
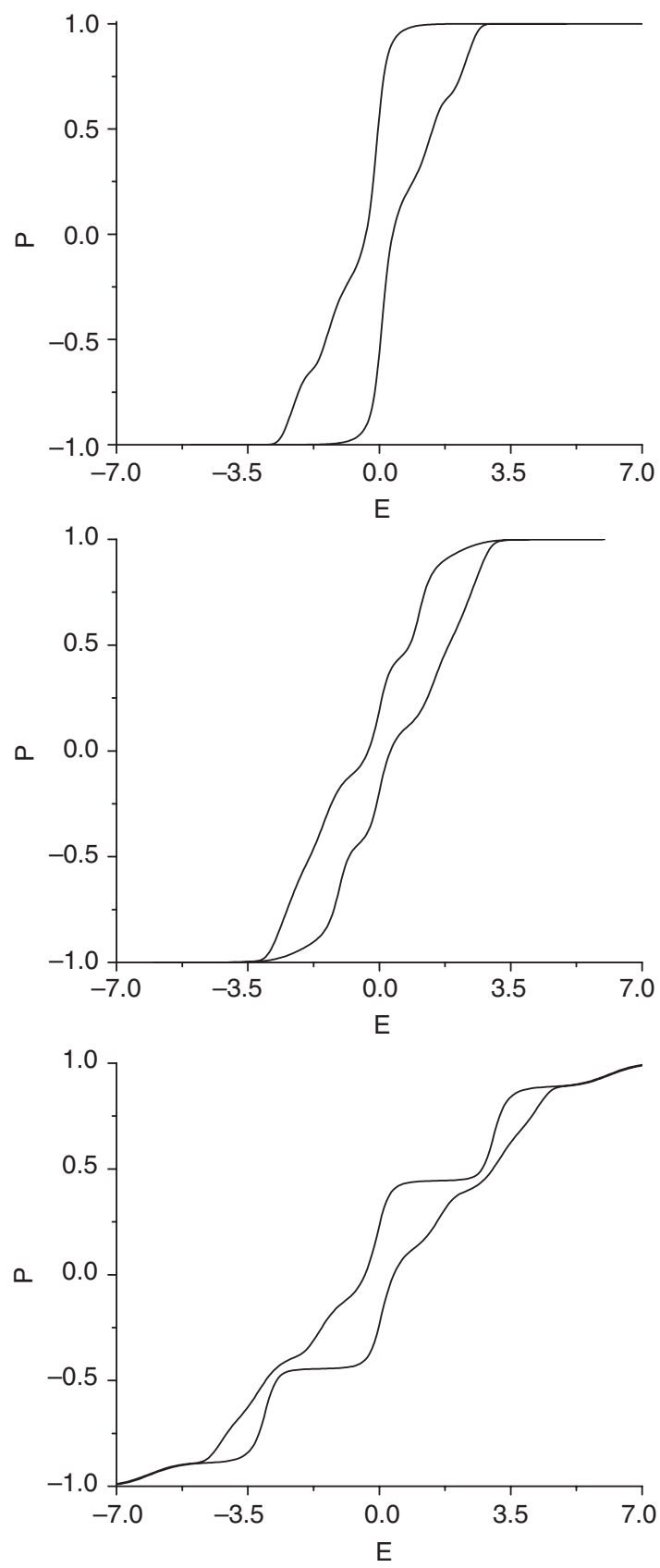

Fig. 5 Tailored series of hysteresis loops for values of $\gamma$, top to bottom, of 1.0, 2.0, and 4.0, for a linear size of the domain of 6 , and for an $\alpha$ value of 1.5 .

tric compound is introduced into the antiferroelectric one, clusters of ferroelectric domains are formed, which increase in size with increasing concentration. The relative size of ferro- and antiferroelectric regions together with the temperature dependence of the relative strength of these interactions explains the different multiple loops along the temperature concentration phase diagram. Systems with competing ferro- and antiferroelectric interactions, such as the ones mentioned above should be characterized by a 

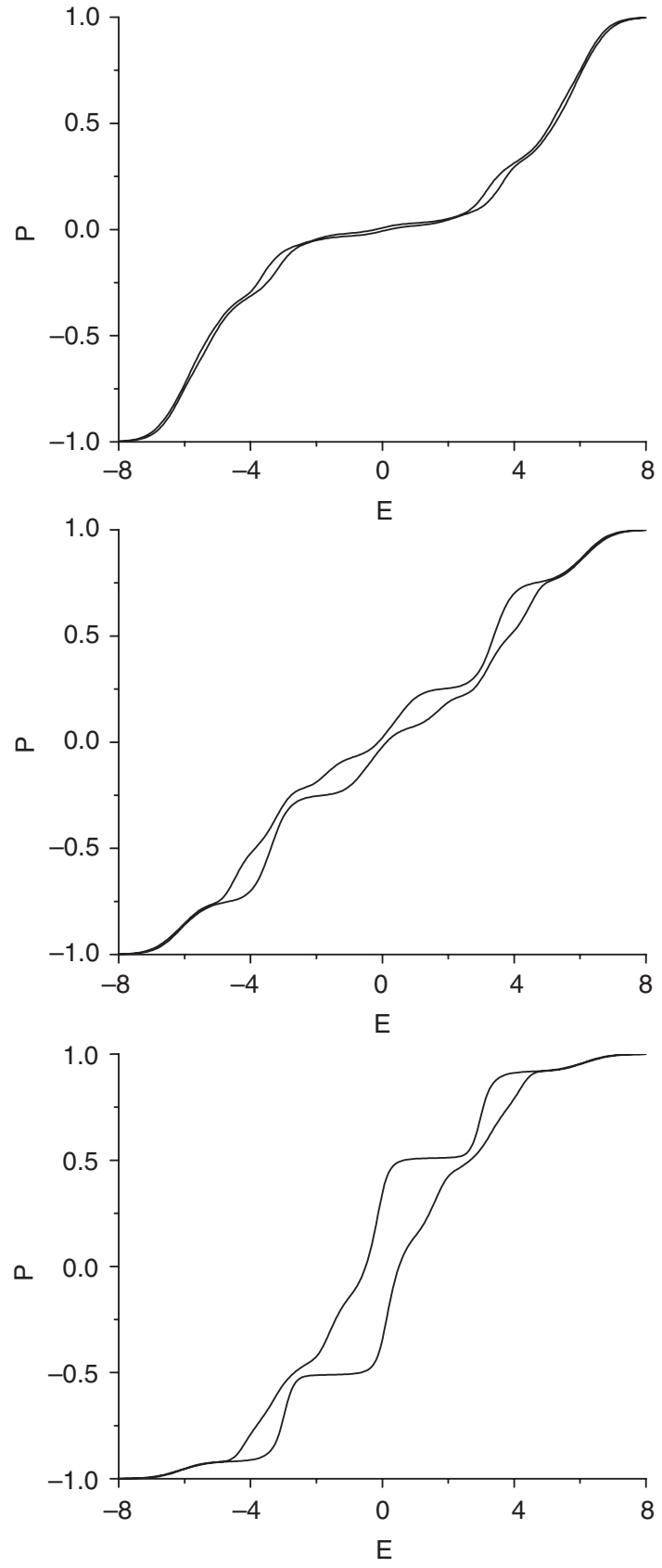

Fig. 6 Tailored series of hysteresis loops for values of linear domain sizes, top to bottom, of 3,4 , and 7 , for an $\alpha$ value of 4.0, and for a $\gamma$ value of 1.5 .
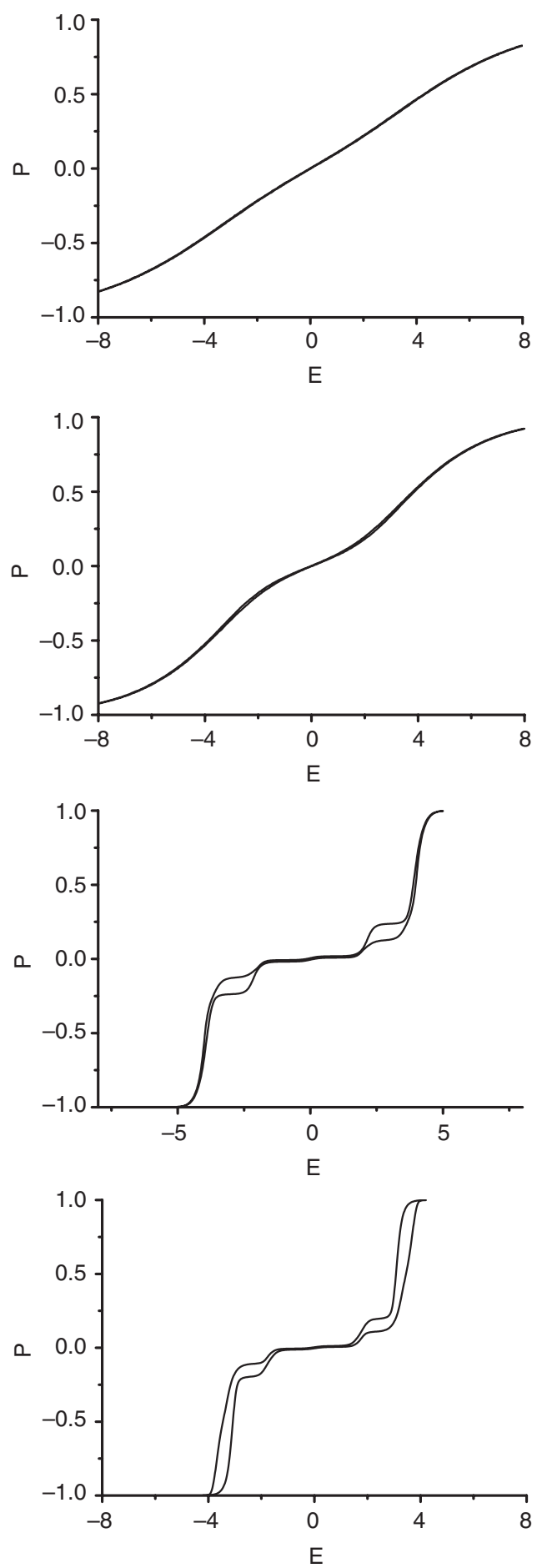

Fig. 7 Series of simulated hysteresis loops showing qualitative agreement to experimental ones. From top to bottom one has $(0.3,3.0,3),(0.4$, $3.0,3),(2.5,3.0,3)$, and $(2.5,2.5,3)$, where each triplet represents $(\alpha, \gamma$, domain size).

() 2005 WILEY-VCH Verlag GmbH \& Co. KGaA, Weinheim 
more random distribution of ferro- and antiferroelectric contributions. In systems with multiple hysteresis loops, phase segregation rules.

\section{Conclusion}

A model introducing the coexistence of ferroelectric and antiferroelectric interactions, common in a variety of materials such as relaxor ferroelectric, proton glass systems or multilayered thin films has been presented. The model is based in the existence of ferroelectric domains with antiferroelectric interfacial coupling. Hysteresis loops with varying domain size and for various temperatures show different degrees of complexity resembling the relative strength of the different interactions.

Hysteresis loops obtained in simulations clearly describe the qualitative features of those appearing in experiment along the temperature-concentration phase diagram of the $\mathrm{BA}_{x} \mathrm{BP}_{1-x}$ mixed crystal. In this way, the ferroelectric, antiferroelectric or mixed character of different regions of the phase diagram becomes clear in the light of ferroelectric domains separated by antiferroelectric interfaces as our comparative study between experiment and simulations of hysteresis loops shows.

Acknowledgements This research has been supported by Fundação para a Ciência e a Tecnologia (grants POCTI/CTM/41574/2001 - Computational Nanophysics (AC) and POCTI/CTM/33501/99 (SLM)).

\section{References}

[1] D. Sherrington and S. Kirkpatrick, Phys. Rev. Lett. 35, 1792 (1975).

[2] P. G. de Gennes, Solid State Commun. 1, 132 (1963).

[3] Y.-Z. Wu, D.-L. Yao, and Z.-Y. Li, J. Appl. Phys. 91, 1482 (2002).

[4] S. Lanceros-Méndez, H. Ebert, G. Schaack, and A. Klöpperpieper, Phys. Rev. B 67, 014109 (2003).

[5] M. Manger, S. Lanceros-Méndez, G. Schaack, and A. Köpperpieper, J. Phys.: Condens. Matter 8, 4617 (1996).

[6] S. Lanceros-Méndez and G. Schaack, Ferroelectrics 226, 107 (1999).

[7] M. Maeda, Ferroelectrics 96, 269 (1989).

[8] M. Maeda and I. Suzuki, Ferroelectrics 108, 351 (1990).

[9] S. Hayase, T. Koshiba, H. Terauchi, M. Maeda, and I. Suzuki, Ferroelectrics 96, 221 (1989).

[10] J. Mano and S. Lanceros-Méndez, J. Appl. Phys. 89, 1844 (2001).

[11] U. Hochli, K. Knorr, and A. Loidl, Adv. Phys. 39, 405 (1990).

[12] J. Hemberger, H. Ries, A. Loidl, and R. Böhmer, Phys. Rev. Lett. 76, 2330 (1996).

[13] T. Yoshida, H. Mashiyama, and T. Mochida, J. Korean Phys. Soc. 35, 1409 (1999).

[14] T. Yoshida, H. Mashiyama, and T. Mochida, J. Phys. Soc. Jpn. 70, 1598 (2001a).

[15] W. Schildkamp and J. Spilker, Z. Kristallogr. 168, 159 (1984).

[16] W. Schildkamp, J. Spilker, and G. Schäfer, Z. Kristallogr. 168, 187 (1984).

[17] T. Yoshida, H. Mashiyama, and T. Mochida, J. Phys. Soc. Jpn. 70, 569 (2001b).

[18] H. J. Rother, J. Albers, A. Klöpperpieper, and H. E. Müser, Jpn. J. Appl. Phys. 24, 384 (1985).

[19] Y.-H. Kim, B.-G. Kim, J.-J. Kim, T. Mochida, and S. Miyajima, J. Phys.: Condens. Matter 8, 6095 (1996).

[20] S. Lanceros-Méndez, J. Mano, and J. Mendes, Ferroelectrics 270, 1457 (2002). 